\title{
Promoter methylation of ITF2, but not APC, is associated with microsatellite instability in two populations of colorectal cancer patients
}

Andrea J. Savio ${ }^{1,2}$, Darshana Daftary ${ }^{2,3}$, Elizabeth Dicks ${ }^{4}$, Daniel D. Buchanan ${ }^{5,6}$, Patrick S. Parfrey ${ }^{4}$, Joanne P. Young ${ }^{7}$, Daniel Weisenberger ${ }^{8}$, Roger C. Green ${ }^{4}$, Steven Gallinger ${ }^{1,2,3,9}$, John R. McLaughlin 2,3,10, Julia A. Knight ${ }^{2,10}$ and Bharati Bapat ${ }^{1,2,11^{*}}$

\begin{abstract}
Background: Aberrant Wnt signaling activation occurs commonly in colorectal carcinogenesis, leading to upregulation of many target genes. APC (adenomatous polyposis coli) is an important component of the $\beta$-catenin destruction complex, which regulates Wnt signaling, and is often mutated in colorectal cancer (CRC). In addition to mutational events, epigenetic changes arise frequently in CRC, specifically, promoter hypermethylation which silences tumor suppressor genes. APC and the Wnt signaling target gene ITF2 (immunoglobulin transcription factor 2) incur hypermethylation in various cancers, however, methylation-dependent regulation of these genes in CRC has not been studied in large, well-characterized patient cohorts. The microsatellite instability (MSI) subtype of CRC, featuring DNA mismatch repair deficiency and often promoter hypermethylation of MutL homolog 1 (MLH1), has a favorable outcome and is characterized by different chemotherapeutic responses than microsatellite stable (MSS) tumors. Other epigenetic events distinguishing these subtypes have not yet been fully elucidated.
\end{abstract}

Methods: Here, we quantify promoter methylation of ITF2 and APC by MethyLight in two case-case studies nested in population-based CRC cohorts from the Ontario Familial Colorectal Cancer Registry $(n=330)$ and the Newfoundland Familial Colorectal Cancer Registry $(n=102)$ comparing MSI status groups.

Results: ITF2 and APC methylation are significantly associated with tumor versus normal state (both $P<1.0 \times 10^{-6}$ ). ITF2 is methylated in $45.8 \%$ of MSI cases and $26.9 \%$ of MSS cases and is significantly associated with MSI in Ontario $(P=0.002)$ and Newfoundland $(P=0.005)$ as well as the MSl-associated feature of $M L H 1$ promoter hypermethylation $\left(P=6.72 \times 10^{-4}\right)$. APC methylation, although tumor-specific, does not show a significant association with tumor subtype, age, gender, or stage, indicating it is a general tumor-specific CRC biomarker.

Conclusions: This study demonstrates, for the first time, MSI-associated ITF2 methylation, and further reveals the subtype-specific epigenetic events modulating Wht signaling in CRC.

Keywords: Colorectal cancer, DNA methylation, Microsatellite instability, Wnt signaling, MethyLight

\footnotetext{
* Correspondence: bapat@lunenfeld.ca

'Department of Laboratory Medicine and Pathobiology, University of

Toronto, Toronto, ON, Canada

${ }^{2}$ Lunenfeld-Tanenbaum Research Institute of Mount Sinai Hospital, Toronto,

ON, Canada

Full list of author information is available at the end of the article
}

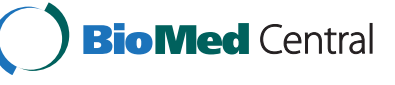

C 2016 Savio et al. Open Access This article is distributed under the terms of the Creative Commons Attribution 4.0 International License (http://creativecommons.org/licenses/by/4.0/), which permits unrestricted use, distribution, and reproduction in any medium, provided you give appropriate credit to the original author(s) and the source, provide a link to the Creative Commons license, and indicate if changes were made. The Creative Commons Public Domain Dedication waiver (http://creativecommons.org/publicdomain/zero/1.0/) applies to the data made available in this article, unless otherwise stated. 


\section{Background}

Colorectal cancer (CRC) is one of the most common cancers in the Western world and is marked by a high mortality rate [1]. Early detection of CRC is the key to improved survival rates [2]. Another factor affecting disease prognosis is CRC subtype [3]. The microsatellite instability (MSI) subtype of CRC accounts for approximately $15 \%$ of colorectal cancers [4]. MSI tumors are distinguished by defects in the DNA mismatch repair system which leads to mutational insertions and deletions in short tandem repeats (microsatellites) of DNA [5]. MSI is most often due to promoter hypermethylation and silencing of the MutL homolog 1 (MLH1) mismatch repair gene. Microsatellite stable (MSS) tumors account for $85 \%$ of CRCs and exhibit chromosomal instability, including numerous chromosomal duplications, deletions and rearrangements [6]. MSI tumors differ from MSS tumors in several ways; MSI CRCs exhibit proximal colonic location, increased lymphocytic infiltration, and poorer response to chemotherapeutic drugs $[7,8]$. MSI CRCs also demonstrate better prognosis at stages I-III, however, some studies suggest poor prognosis at stage IV, though metastatic MSI cases are rare $[7,9]$. A third CRC subtype, the CpG island methylator phenotype (CIMP), is characterized by widespread DNA hypermethylation of CpG-rich promoter islands. CIMP can exist concurrently with either the MSI or MSS phenotype, though it is more frequently found in tandem with MSI and MLH1 hypermethylation [10]. The prognostic significance of CIMP is currently undefined and may be modified by MSI status, presence of $B R A F$ mutation, tumor stage, or other factors [11-13]. Recently, a classification system for further subtyping of CRC has been proposed, consisting of four subtypes [14]. One subtype consists mostly of MSI cases, while the other three are able to categorize the remainder of cases by Wnt signaling activation, metabolic dysregulation, or mesenchymal activation.

The vast majority (up to $94 \%$ ) of CRCs feature dysregulation in the Wnt signaling pathway [15]. Wnt signaling is important in normal development, cell growth and proliferation, but when inappropriately activated may also lead to tumor initiation and development [16]. In canonical Wnt signaling, $\beta$-catenin accumulates within the cell, enters the nucleus and activates transcription of target genes, such as c-Myc and ITF2 (immunoglobulin transcription factor 2) [17, 18]. ITF2 is also known as transcription factor 4 (TCF4). In the absence of Wnt signaling, a $\beta$-catenin destruction complex including adenomatous polyposis coli (APC) targets $\beta$-catenin for ubiquitination followed by proteasomal degradation $[17,18]$. In many cases of CRC, the $A P C$ gene is mutated, rendering it incapable of binding to $\beta$-catenin, which leads to $\beta$-catenin accumulation followed by its nuclear translocation and subsequent activation of downstream target genes [18].

Evidence for DNA methylation of the $A P C$ promoter has been found in CRC. However, to what extent $A P C$ methylation plays a role in colorectal carcinogenesis is unclear, as a broad range of methylation levels has been found in the literature, from 11 up to $63 \%$ of tumors methylated [19-23]. Conflicting reports exist regarding the extent of $A P C$ methylation in MSI CRCs. Some small-scale studies (MSI $n \leq 29$ ) have suggested that $A P C$ methylation may be associated with the MSI subtype, but others show no significant difference [21-27]. Still another study has found $A P C$ methylation to be inversely correlated with CIMP but not MSI [28].

The role of ITF2, a Wnt signaling target gene, is less understood in CRC. It is a target of Wnt signaling and is overexpressed in colon cancers with Wnt dysregulation [29]. Its expression was reported elevated in some cancers with aberrant Wnt signaling activation but decreased in others [30, 31]. Among gastrointestinal malignancies, ITF2 methylation has been reported in gastric cancer, but its methylation status has not been investigated in CRC [32,33].

Our group has previously demonstrated associations between the methylation status of key Wnt signaling pathway regulatory genes and CRC subtype including the extracellular Wnt antagonists DKK1 and SFRP1 as well as Wnt5a which is involved in non-canonical Wnt activity [34, 35].

In this study, we have examined the role of $A P C$ and ITF2 methylation in two nested case-case studies in CRC cohorts. These patients were recruited from two distinct Canadian populations and the case groups were stratified by their MSI status.

\section{Methods}

\section{Study participants}

Participants of this study were population-based primary CRC cases recruited through the Ontario Familial Colorectal Cancer Registry (OFCCR) and Newfoundland Familial Colorectal Cancer Registry (NFCCR). Procedures for patient accrual, biospecimen collection and data collection for the OFCCR and NFCCR have been previously described [36, 37]. Briefly, Ontario residents between the ages of 20 and 74 diagnosed with pathology-confirmed primary CRC between 1997 and 2000 were eligible for recruitment. Familial adenomatous polyposis cases were excluded and in the current study non-white patients were also excluded due to the high prevalence of self-reported Caucasians in the study (92.5\%). A total of 1168 participants have been analyzed for MSI status (see Molecular analysis below) of which 184 are MSI high (MSI-H). 165 of these MSI$\mathrm{H}$ cases had available DNA of high quality. A matched case-case selection strategy was utilized to select 165 
patients with MSS tumors to match 165 patients with MSI-H tumors by sex, stage at diagnosis and age quartile. The 165 MSS tumors were selected from a total of 384 MSS tumors available at the time this study was undertaken. Population-based recruitment by the NFCCR was similar to the OFCCR, with a recruitment period from 1999 to 2003 of cases from provincial tumor registries [37]. For the NFCCR, proxy consent from living family members was obtained for deceased patients leading to a high frequency of late-stage patients. These tumor samples were not utilized in order to maintain similar patient age and tumor stage between the Ontario and Newfoundland populations. 102 tumor samples from 696 total CRC cases were chosen from probands of the NFCCR, 51 of which were MSI-H, matched to 51 MSS cases by sex, stage at diagnosis and age quartile. Overall survival status, along with other patient clinicopathological features, is described in Table 1. Recurrence data was not available for all cases used in this study, thus was not included in analysis. DNA from normal colonic mucosa was also available for all patients. Of the 330 OFCCR and 102 NFCCR patients' tumor samples utilized, 47 were selected randomly for methylation analysis of normal adjacent tissue. Patient data was obtained through protocols approved by the Research Ethics Boards of Mount Sinai Hospital, the University of Toronto and Memorial University of Newfoundland. All patients or their proxies provided informed consent.

\section{Molecular analysis}

DNA used to assess MSI status was extracted from archival paraffin-embedded tumors microdissected to enrich for tumor cells. MSI status was assessed using National Cancer Institute guidelines using four or more of the following markers: ACTC, BAT-25, BAT-26, BAT-40, BAT-34C4, D10S197, D18S55, D17S250, D5S346 and MYC-L. The numbers of positive markers used to define MSI status are: MSI high (MSI-H), $\geq 30 \%$ unstable markers; MSI low (MSI-L), 1-29 \% unstable markers; MSS, 0 \% unstable markers [38]. Tumors with MSI-L status were not included in this study.

Somatic T > A mutation of nucleotide 1799 in the BRAF gene leading to the V600E mutation was determined by allele-specific PCR as described previously [34].

Immunohistochemistry was used to determine presence of the mismatch repair proteins MLH1, MSH2, MSH6 and PMS2. Protein staining was classified as either present, absent, or inconclusive. Tumors without positive staining for any of these proteins were defined as mismatch repair deficient, as described previously [39].

\section{MethyLight analysis}

The sensitive, semi-quantitative high-throughput MethyLight assay was used to analyze the methylation of $A P C$ and ITF2 in tumor and normal colonic DNA of CRC patients. DNA was treated with sodium bisulfite prior to MethyLight according to protocol using the EZ DNA Methylation Gold Kit (Zymo Research Corp, Orange, $\mathrm{CA})$. Primers and probe were used to amplify a region within the $\mathrm{CpG}$ island of promoter $1 \mathrm{~A}$ of $A P C$. Forward primer: 5'-GAACCAAAACGCTCCCCAT-3'. Probe: 5' CCCGTCGAAAACCCGCCGATTA-3'. Reverse primer: 5'-TTATATGTCGgTtACGTGCGTTTATAT-3'. Primers and probe were designed within the promoter region of ITF2. Forward primer: 5'-GAAGCGGTAATACGAATAA GAGC-3'. Probe: 5'-ATTCCCGAAACCGAAATCGTTC GCAAACC-3'. Reverse primer: 5' - AACTATTCTCGAAT AAACGTCGC-3'. Alu-C4 was also amplified to normalize the DNA input. Forward primer: 5'-GGTTAGGTATAGT GGTTTATATTTGTAATT-3'. Probe: 5'-CCTACCTTAA CCTCCC-3'. Reverse primer: 5'-ATTAACTAAACTAATC TTAAACTCCTA-3'. Probes contained a $5^{\prime}$ fluorescent reporter dye and a 3' quencher dye. Samples were analyzed using the ABI 7500 RT-PCR thermocycler in 96-well plates as previously described [40]. APC, ITF2 and Alu-C4 were also amplified in exogenously methylated CpGenome DNA (Millipore, Billerica, MA). The percent methylated reference (PMR) was calculated to assess the methylation using the formula: (Gene of Interest/Alu-C4) sample/(Gene of Interest/Alu-C4) ${ }_{\mathrm{CpGenome}} \mathrm{x} 100 \%$. In order to ensure that DNA quality was adequate, samples with an Alu-C4 threshold cycle greater than 22 were deemed poor quality and reanalyzed or removed from the study [41].

MLH1 methylation status was assessed by MethyLight as described previously, with positive methylation defined as $\mathrm{PMR} \geq 10 \%$ [42]. CIMP status was determined using the Weisenberger panel of markers, described previously [43]. Briefly, MethyLight was used to assess a 5-gene signature consisting of CACNA1G, IGF2, NEUROG1, RUNX3 and SOCS1. Tumors were classified as CIMP if 3 or more of 5 genes had PMR $\geq 10 \%$ and non-CIMP if 2 or fewer genes had PMR $\geq 10 \%$. CIMP status was available for a subset of Ontario cases (285 of 330) and unavailable for Newfoundland cases.

\section{Statistical analysis}

Comparison of the methylation status of matched tumor and normal DNA samples was performed using McNemar's test. Results were considered statistically significant if two-sided $P<0.05$. Pearson's chi-square test was used to measure associations between clinicopathological variables and ITF2 and APC methylation in tumor DNA. Bonferroni correction was used to account for multiple comparisons. All analyses were performed using PASW Statistics 21 (SPSS Inc., Chicago, IL). 
Table 1 Clinicopathological features of primary colorectal carcinomas of patients from Ontario and Newfoundland

\begin{tabular}{|c|c|c|c|c|}
\hline & \multicolumn{4}{|c|}{ No. of cases (\%) } \\
\hline & \multicolumn{2}{|l|}{ Ontario } & \multicolumn{2}{|c|}{ Newfoundland } \\
\hline & MSS & MSI-H & $\overline{M S S}$ & MSI-H \\
\hline $\begin{array}{l}\text { Cases of primary } \\
\text { colorectal carcinoma }\end{array}$ & 165 & 165 & 51 & 51 \\
\hline Mean age $\left( \pm \mathrm{SD}^{\mathrm{a}}\right)$ & $59.9(9.3)$ & $60.1(9.8)$ & $58.3(10.2)$ & $58.4(10.2)$ \\
\hline \multicolumn{5}{|l|}{ Age } \\
\hline$<50$ & $19(11.5)$ & $28(17.0)$ & $11(21.6)$ & $10(19.6)$ \\
\hline $50+$ & $146(88.5)$ & $137(83.0)$ & $40(78.4)$ & $41(80.4)$ \\
\hline \multicolumn{5}{|l|}{ Sex } \\
\hline Male & $74(44.8)$ & $74(44.8)$ & $26(51.0)$ & $26(51.0)$ \\
\hline Female & $91(55.2)$ & $91(55.2)$ & $25(49.0)$ & $25(49.0)$ \\
\hline \multicolumn{5}{|l|}{ TNM Stage } \\
\hline 1 & $38(23.0)$ & $38(23.0)$ & $12(23.5)$ & 11 (21.6) \\
\hline 2 & $84(50.9)$ & 85 (51.5) & $26(51.0)$ & $27(52.9)$ \\
\hline 3 & $34(20.6)$ & 39 (23.6) & $10(19.6)$ & $12(23.5)$ \\
\hline 4 & $9(5.5)$ & $3(1.8)$ & $3(5.9)$ & $1(2.0)$ \\
\hline \multicolumn{5}{|l|}{ Histological Grade } \\
\hline Low & $16(9.7)$ & $10(6.1)$ & $4(7.8)$ & $7(13.7)$ \\
\hline Moderate & $123(74.5)$ & $45(27.3)$ & $37(72.6)$ & $36(70.6)$ \\
\hline High & $13(7.9)$ & $20(12.1)$ & $8(15.7)$ & $8(15.7)$ \\
\hline Unavailable & $13(7.9)$ & $90(54.5)$ & $2(3.9)$ & \\
\hline \multicolumn{5}{|l|}{ Location $^{\mathrm{b}}$} \\
\hline Distal & $108(65.5)$ & $15(9.1)$ & $37(72.5)$ & $9(17.6)$ \\
\hline Proximal & $51(30.9)$ & $63(38.2)$ & $14(27.5)$ & $42(82.4)$ \\
\hline Unavailable & $6(3.6)$ & $87(52.7)$ & & \\
\hline \multicolumn{5}{|l|}{ Histological Type ${ }^{c}$} \\
\hline Non-Mucinous & $143(86.7)$ & $107(64.8)$ & $46(90.2)$ & $42(82.4)$ \\
\hline Mucinous & 19 (11.5) & $53(32.1)$ & $5(9.8)$ & $9(17.6)$ \\
\hline Unavailable & $3(1.8)$ & $5(3.0)$ & & \\
\hline \multicolumn{5}{|l|}{ MMR Protein Status } \\
\hline Intact & $148(89.7)$ & $26(15.8)$ & $50(98.0)$ & $4(7.8)$ \\
\hline Deficient & $4(2.4)$ & $136(82.4)$ & $0(0.0)$ & $46(90.2)$ \\
\hline Unavailable & $13(7.9)$ & $3(1.8)$ & $1(2.0)$ & $1(2.0)$ \\
\hline \multicolumn{5}{|c|}{ MMR Germline Mutation } \\
\hline No & $164(99.4)$ & $124(75.2)$ & $51(100.0)$ & $39(76.5)$ \\
\hline Yes & $1(0.6)$ & $41(24.8)$ & $0(0.0)$ & $12(23.5)$ \\
\hline \multicolumn{5}{|l|}{ MLH1 Methylation } \\
\hline Unmethylated & $159(96.4)$ & $87(52.7)$ & $36(70.6)$ & $23(45.1)$ \\
\hline Methylated & $5(3.0)$ & $78(47.3)$ & $1(2.0)$ & $28(54.9)$ \\
\hline Unavailable & $1(0.6)$ & & $14(27.4)$ & \\
\hline \multicolumn{5}{|l|}{ BRAF V600E Mutation } \\
\hline No & $146(88.5)$ & $95(57.6)$ & $46(90.2)$ & $25(49.0)$ \\
\hline Yes & $15(9.1)$ & $66(40.0)$ & $2(3.9)$ & $20(39.2)$ \\
\hline Unavailable & $4(2.4)$ & $4(2.4)$ & $3(5.9)$ & $6(11.8)$ \\
\hline
\end{tabular}

Table 1 Clinicopathological features of primary colorectal carcinomas of patients from Ontario and Newfoundland (Continued)

\begin{tabular}{lllll}
\hline CIMP Status & & & & \\
Negative & $133(80.6)$ & $79(47.9)$ & & \\
Positive & $10(6.1)$ & $63(38.2)$ & & \\
Unavailable & $22(13.3)$ & $23(13.9)$ & $51(100.0)$ & $51(100.0)$ \\
Survival Status & & & & \\
Alive & $100(60.6)$ & $99(60.0)$ & $45(88.2)$ & $49(96.1)$ \\
Deceased & $65(39.4)$ & $66(40.0)$ & $6(11.8)$ & $2(3.9)$ \\
ITF2 Methylation & & & & \\
Unmethylated & $122(73.9)$ & $95(57.6)$ & $36(70.6)$ & $22(43.1)$ \\
Methylated & $43(26.1)$ & $70(42.4)$ & $15(29.4)$ & $29(56.9)$ \\
APC Methylation & & & & \\
Unmethylated & $112(67.9)$ & $103(67.9)$ & $33(64.7)$ & $28(54.9)$ \\
Methylated & $53(32.1)$ & $62(37.6)$ & $18(35.3)$ & $23(45.1)$ \\
\hline
\end{tabular}

${ }^{\mathrm{a} S t a n d a r d}$ Deviation

${ }^{b}$ Proximal tumor location includes lesions up to and including the splenic flexure

${ }^{c}$ Mucinous histology includes the presence of any mucin within the tumor stroma

\section{Results}

ITF2 promoter methylation in CRC tumors and normal colonic mucosa

Patient clinicopathological features are shown in Table 1 for MSI-H and MSS cases for the Ontario and Newfoundland populations. We quantified promoter methylation of ITF2 using MethyLight in CRC tumors from Ontario and Newfoundland. To quantify ITF2 promoter methylation levels in both normal mucosa and CRC tumor tissue we tested 47 randomly selected normal-tumor pairs from Ontario and Newfoundland CRC cases, of which 12 were MSI-H and 35 were MSS. The mean PMR was $8.8 \%$ in tumor DNA and $1.6 \%$ in normal colonic DNA. Methylation levels ranged from $0-31.1 \%$ in tumor and $0-15.6 \%$ in normal colonic DNA. A PMR cut-off of $10 \%$ was used to dichotomize methylated and unmethylated samples. McNemar's test comparing methylation above this cut-off in tumor and normal tissues in CRC patients determined tumor methylation of ITF2 to be significantly higher than normal colonic mucosa methylation $\left(P<1.0 \times 10^{-6}\right)$. For ITF2 promoter methylation, comparable methylation levels were seen in CRC tumors between the two populations, comprised of 165 MSI-H and 165 MSS cases from Ontario and 51 MSI-H and 51 MSS cases from Newfoundland. In the Ontario cases the mean PMR for all 330 cases was $8.5 \%$. Methylation values ranged from $0-57.2 \%$. Using the PMR cut-off of $10 \%$, $34.2 \%(113 / 330)$ of cases were considered positively methylated in the Ontario cohort. In Newfoundland cases the mean PMR for all 102 cases was $15.4 \%$. Methylation 
values ranged from 0-95.4\%. Using the PMR cut-off of $10 \%, 43.1 \%(44 / 102)$ of cases were considered positively methylated in the Newfoundland cohort.

\section{APC promoter methylation in CRC tumors and normal colonic mucosa}

We quantified methylation of the $A P C$ promoter region in tumor and matched normal colonic mucosa of 47 randomly selected patients from both Ontario and Newfoundland. Mean methylation was $16.1 \%$ in tumor DNA and $2.6 \%$ in normal colon. Methylation values ranged from $0-60.2 \%$ for tumor samples and from $0-11.9 \%$ for normal samples. A PMR cut-off of $10 \%$ was used to dichotomize methylated and unmethylated samples. McNemar's test comparing methylation above this cut-off in tumor and normal tissues in CRC patients determined tumor methylation of $A P C$ to be significantly higher than normal colonic mucosa methylation $\left(P<1.0 \times 10^{-6}\right)$.

For APC promoter methylation, comparable methylation levels were seen in CRC tumors between the two populations. The mean PMR in Ontario was $11.5 \%$. Methylation values ranged from 0-92.2 \%. For the Newfoundland samples, the mean PMR was $13.9 \%$. Methylation values ranged from 0-70.5\%. Using the PMR cut-off of $10 \%, 34.8 \%$ of tumors (115/330) were methylated in the Ontario cohort and $40.2 \%$ of tumors $(41 / 102)$ were methylated in the Newfoundland cohort.

ITF2 methylation and clinicopathological features, including MSI subtype, in two distinct CRC cohorts

We examined whether methylation of ITF2 in tumor DNA was associated with patient clinicopathological features. Methylation status was compared by Pearson's chi-square test between MSI-H and MSS cases. In Ontario $26.1 \%(43 / 165)$ of MSS cases compared to $42.4 \%$ (70/165) of MSI-H cases were methylated, with an odds ratio (OR) of 2.09 [95 \% confidence interval (CI) 1.313.33 , and $P=0.002$. Similarly in Newfoundland $29.4 \%$ $(15 / 51)$ of MSS cases and $56.9 \%(29 / 51)$ of MSI-H cases were methylated with OR of 3.16 (95\% CI 1.40-7.17), and $P=0.005$. Ontario and Newfoundland data was then pooled for further analysis.

Due to our selection strategy and the association between MSI and ITF2 methylation, further clinicopathological associations were analyzed separately for MSI-H and MSS cases, shown in Table 2. There was a significant association between ITF2 promoter methylation with $M L H 1$ promoter methylation in MSI-H cases, $P=$ $6.72 \times 10^{-4}$, OR $1.88(1.10-3.68)$. There was also a trend towards an association between ITF2 methylation and female gender in MSI-H cases, and ITF2 methylation and CIMP in MSS cases, but this was not significant using a conservative $p$-value adjusted for multiple comparisons. No other significant associations were found for either MSS or MSI-H cases between ITF2 methylation and early age of onset ( $<50$ years), stage, grade, tumor location, histological type, MMR protein status, MMR germline mutation, BRAF V600E mutation, or survival status. Stagespecific survival was also performed, to account for potential differences in early stage survival compared to stage IV in MSI-H cases (data not shown). There was a trend toward higher overall survival in stage I MSI-H cases with ITF2 methylation, but results were not significant after correction for multiple comparisons.

\section{APC methylation and clinicopathological features, including MSI subtype, in two distinct CRC cohorts} We examined whether methylation of $A P C$ in tumor DNA was associated with patient clinicopathological features in both cohorts. Methylation status was compared by Pearson's chi-square test between MSI-H and MSS cases. In Ontario $32.1 \%(53 / 165)$ of MSS cases were methylated while $37.6 \%(62 / 165)$ of MSI-H cases were methylated with an OR (95\% CI) of $1.27(0.81-2.00)$, and $P=0.298$. Similarly in Newfoundland $35.3 \%(18 / 51)$ of MSS cases were methylated and $45.1 \%(23 / 51)$ of MSI-H cases methylated with OR (95\% CI) of 1.51 (0.68-3.34), and $P=0.313$.

We examined whether APC methylation was associated with patient clinicopathological features in pooled CRC cases from Ontario and Newfoundland. Results are shown in Table 3. Methylation of APC was not found to be associated with early age of onset $(<50$ years), sex, stage, grade, tumor location, histological type, CIMP status, MMR protein status, MMR germline mutation, $M L H 1$ methylation, BRAF V600E mutation, or survival status.

\section{Discussion}

Understanding the genetic and epigenetic differences amongst colorectal cancer subtypes is essential, as CRC subtypes differ in their treatment options and offer distinct survival outcomes. The Wnt signaling pathway is dysregulated in a majority of colorectal tumors and can be altered at the extracellular, intracellular and gene target level [15, 34, 35]. We have shown that these changes to the Wnt pathway also differ based upon microsatellite instability status. We quantified the methylation status of the ITF2 and APC promoter CpG islands in a nested case-case study in two cohorts of colorectal carcinoma from two different populations, comparing cases by MSI status. We have demonstrated that the ITF2 promoter is hypermethylated in tumor tissues compared with matched normal mucosa, and further, MSI-H tumors are more likely to incur promoter methylation compared with MSS tumors. ITF2 promoter methylation was also significantly associated with $M L H 1$ promoter methylation, a common occurrence in MSI-H tumors. Conversely, we found that $A P C$, an important intracellular regulator of Wnt signaling 
Table 2 Associations between ITF2 methylation and clinicopathological features in tumor DNA. Analysis of 216 MSI-H and 216 MSS CRC patients from Ontario and Newfoundland

\begin{tabular}{|c|c|c|c|c|c|c|c|c|}
\hline & \multicolumn{4}{|l|}{ MSS $(n=216)$} & \multicolumn{4}{|l|}{ MSI-H $(n=216)$} \\
\hline & $\begin{array}{l}\text { Unmethylated } \\
(\%)\end{array}$ & $\begin{array}{l}\text { Methylated } \\
(\%)\end{array}$ & OR $(95 \% \mathrm{Cl})^{\mathrm{a}}$ & $P$-value & $\begin{array}{l}\text { Unmethylated } \\
(\%)\end{array}$ & $\begin{array}{l}\text { Methylated } \\
(\%)\end{array}$ & OR $(95 \% C l)^{a}$ & $P$-value \\
\hline \multicolumn{9}{|l|}{ Age } \\
\hline$<50$ & $24(15.2)$ & $6(10.3)$ & $1.552(0.600-4.014)$ & 0.361 & $24(20.5)$ & $14(14.1)$ & $1.567(0.761-3.225)$ & 0.220 \\
\hline $50+$ & $134(84.8)$ & $52(89.7)$ & & & $93(79.5)$ & $85(85.9)$ & & \\
\hline \multicolumn{9}{|l|}{ Sex } \\
\hline Male & $73(46.2)$ & $26(44.8)$ & $1.057(0.577-1.935)$ & 0.857 & $64(54.7)$ & $36(36.4)$ & $2.113(1.222-3.655)$ & 0.007 \\
\hline Female & $85(53.8)$ & $32(55.2)$ & & & $53(45.3)$ & $63(63.6)$ & & \\
\hline \multicolumn{9}{|l|}{ TNM Stage $^{b}$} \\
\hline 1 & $36(22.8)$ & $14(24.1)$ & $0.720(0.606-0.856)$ & 0.193 & $31(26.5)$ & $18(18.2)$ & $5.167(0.499-53.450)$ & 0.321 \\
\hline 2 & $78(49.4)$ & $32(55.2)$ & & & $57(48.7)$ & $55(55.6)$ & & \\
\hline 3 & $32(20.3)$ & $12(20.7)$ & & & $28(23.9)$ & $23(23.2)$ & & \\
\hline 4 & $12(7.6)$ & $0(0.0)$ & & & $1(0.9)$ & $3(3.0)$ & & \\
\hline \multicolumn{9}{|l|}{ Histological Grade ${ }^{b}$} \\
\hline Low & $16(10.8)$ & $4(7.5)$ & $2.000(0.482-8.295)$ & 0.624 & $9(13.8)$ & $8(13.1)$ & $0.728(0.215-2.459)$ & 0.514 \\
\hline Moderate & $118(79.7)$ & $42(79.2)$ & & & $39(60.0)$ & $42(68.9)$ & & \\
\hline High & $14(9.5)$ & $7(13.2)$ & & & $17(26.2)$ & $11(18.0)$ & & \\
\hline \multicolumn{9}{|l|}{ Location $^{c}$} \\
\hline Proximal & $58(37.9)$ & $29(50.9)$ & 0.589 (0.319-1.089) & 0.090 & 60 (89.6) & $54(87.1)$ & $1.270(0.432-3.735)$ & 0.664 \\
\hline Distal & $95(62.1)$ & $28(49.1)$ & & & $7(10.4)$ & $8(12.9)$ & & \\
\hline \multicolumn{9}{|l|}{ Histological Type ${ }^{d}$} \\
\hline Non-Mucinous & $140(90.3)$ & $49(84.5)$ & $1.714(0.705-4.167)$ & 0.230 & $76(66.7)$ & $73(75.3)$ & $0.658(0.360-1.202)$ & 0.172 \\
\hline Mucinous & $15(9.7)$ & $9(15.5)$ & & & $38(33.3)$ & $24(24.7)$ & & \\
\hline \multicolumn{9}{|c|}{ MMR Protein Status } \\
\hline Intact & $143(97.9)$ & $55(98.2)$ & $0.867(0.088-8.511)$ & 0.902 & $21(18.6)$ & $9(9.1)$ & $2.283(0.992-5.251)$ & 0.048 \\
\hline Deficient & $3(2.1)$ & $1(1.8)$ & & & $92(81.4)$ & $90(90.9)$ & & \\
\hline \multicolumn{9}{|c|}{ MMR Germline Mutation } \\
\hline No & $157(99.4)$ & $58(100.0)$ & $0.730(0.673-0.792)$ & 0.544 & $89(76.1)$ & $74(74.7)$ & $1.074(0.577-1.999)$ & 0.822 \\
\hline Yes & $1(0.6)$ & $0(0.0)$ & & & $28(23.9)$ & $25(25.3)$ & & \\
\hline \multicolumn{9}{|l|}{ MLH1 Methylation } \\
\hline Unmethylated & $143(96.0)$ & $52(100.0)$ & $0.733(0.674-0.798)$ & 0.142 & $68(58.1)$ & $42(42.4)$ & $1.883(1.095-3.238)$ & $6.72 \times 10^{-4}$ \\
\hline Methylated & $6(4.0)$ & $0(0.0)$ & & & $49(41.9)$ & $57(57.6)$ & & \\
\hline \multicolumn{9}{|c|}{ BRAF V600E Mutation } \\
\hline No & $141(92.2)$ & $51(91.1)$ & $1.152(0.387-3.431)$ & 0.799 & $70(63.1)$ & $50(52.6)$ & $1.537(0.088-2.683)$ & 0.130 \\
\hline Yes & $12(7.8)$ & $5(8.9)$ & & & $41(36.9)$ & $45(47.4)$ & & \\
\hline \multicolumn{9}{|l|}{ CIMP Status } \\
\hline Negative & $100(96.2)$ & $33(84.6)$ & $4.545(1.208-17.100)$ & 0.016 & $50(61.7)$ & $29(47.5)$ & $1.780(0.908-3.489)$ & 0.092 \\
\hline Positive & $4(3.8)$ & $6(15.4)$ & & & $31(38.3)$ & $32(52.5)$ & & \\
\hline \multicolumn{9}{|l|}{ Survival Status } \\
\hline Alive & $110(69.6)$ & $35(60.3)$ & $1.475(0.790-2.755)$ & 0.221 & $78(66.7)$ & $70(70.7)$ & $0.829(0.464-1.478)$ & 0.524 \\
\hline Deceased & $48(30.4)$ & 23 (39.7) & & & $39(33.3)$ & $29(29.3)$ & & \\
\hline
\end{tabular}

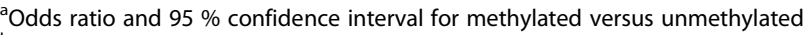

${ }^{\mathrm{b}} \mathrm{OR}$ and $95 \% \mathrm{Cl}$ given for lowest stage/grade versus highest stage/grade

'Proximal tumor location includes lesions up to and including the splenic flexure

${ }^{d}$ Mucinous histology includes the presence of any mucin within the tumor stroma 
Table 3 Associations between APC methylation and clinicopathological features in tumor DNA. Analysis of 216 MSI-H and 216 MSS CRC patients from Ontario and Newfoundland

\begin{tabular}{|c|c|c|c|c|c|c|c|c|}
\hline & \multicolumn{4}{|l|}{ MSS $(n=216)$} & \multicolumn{4}{|l|}{ MSI-H $(n=216)$} \\
\hline & $\begin{array}{l}\text { Unmethylated } \\
(\%)\end{array}$ & $\begin{array}{l}\text { Methylated } \\
\text { (\%) }\end{array}$ & OR $(95 \% \mathrm{Cl})^{\mathrm{a}}$ & $P$-value & $\begin{array}{l}\text { Unmethylated } \\
(\%)\end{array}$ & $\begin{array}{l}\text { Methylated } \\
(\%)\end{array}$ & OR $(95 \% \mathrm{Cl})^{\mathrm{a}}$ & $P$-value \\
\hline \multicolumn{9}{|l|}{ Age } \\
\hline$<50$ & $20(13.8)$ & $10(14.1)$ & $0.976(0.431-2.213)$ & 0.954 & $23(17.6)$ & $15(17.9)$ & $0.994(0.485-2.035)$ & 0.986 \\
\hline $50+$ & $125(86.2)$ & $61(85.9)$ & & & $108(82.4)$ & $70(82.4)$ & & \\
\hline \multicolumn{9}{|l|}{ Sex } \\
\hline Male & $64(44.1)$ & $35(49.3)$ & $0.813(0.460-1.436)$ & 0.475 & $59(45.0)$ & $41(48.2)$ & $0.879(0.509-1.520)$ & 0.645 \\
\hline Female & $81(55.9)$ & $36(50.7)$ & & & $72(55.0)$ & $44(51.8)$ & & \\
\hline \multicolumn{9}{|l|}{ TNM Stage $^{\mathrm{b}}$} \\
\hline 1 & 27 (18.6) & $23(32.4)$ & $0.391(0.095-1.619)$ & 0.147 & $32(24.4)$ & $17(20.0)$ & $0.653(0.533-0.801)$ & 0.306 \\
\hline 2 & 79 (54.5) & $31(43.7)$ & & & $64(48.9)$ & $48(56.5)$ & & \\
\hline 3 & $30(20.7)$ & $14(19.7)$ & & & $31(23.7)$ & $20(23.5)$ & & \\
\hline 4 & $9(6.2)$ & $3(4.2)$ & & & $4(3.1)$ & $0(0.0)$ & & \\
\hline \multicolumn{9}{|c|}{ Histological Grade } \\
\hline Low & $12(8.9)$ & $8(12.1)$ & $0.600(0.163-2.207)$ & 0.724 & $8(11.0)$ & $9(17.0)$ & $0.770(0.230-2.578)$ & 0.467 \\
\hline Moderate & $108(80.0)$ & $52(78.8)$ & & & $50(68.5)$ & $31(58.5)$ & & \\
\hline High & $15(11.1)$ & $6(9.1)$ & & & $15(20.5)$ & $13(24.5)$ & & \\
\hline \multicolumn{9}{|l|}{ Location $^{c}$} \\
\hline Proximal & $62(44.0)$ & $25(36.2)$ & $1.381(0.763-2.499)$ & 0.285 & $63(85.1)$ & $51(92.7)$ & $0.449(0.135-1.495)$ & 0.183 \\
\hline Distal & $79(56.0)$ & $44(63.8)$ & & & $11(14.9)$ & $4(7.3)$ & & \\
\hline \multicolumn{9}{|l|}{ Histological Type ${ }^{d}$} \\
\hline Non-Mucinous & $129(89.6)$ & $60(87.0)$ & $1.290(0.534-3.114)$ & 0.570 & $87(68.5)$ & $62(73.8)$ & $0.772(0.418-1.426)$ & 0.408 \\
\hline Mucinous & $15(10.4)$ & $9(13.0)$ & & & $40(31.5)$ & $22(26.2)$ & & \\
\hline \multicolumn{9}{|c|}{ MMR Protein Status } \\
\hline Intact & $132(97.8)$ & $66(98.5)$ & $0.668(0.068-6.533)$ & 0.726 & $21(16.3)$ & $9(10.8)$ & $1.599(0.694-3.685)$ & 0.268 \\
\hline Deficient & $3(2.2)$ & $1(1.5)$ & & & $108(83.7)$ & $74(89.2)$ & & \\
\hline \multicolumn{9}{|c|}{ MMR Germline Mutation } \\
\hline No & $144(99.3)$ & $71(100.0)$ & $0.670(0.610-0.736)$ & 0.483 & $104(79.4)$ & $59(69.4)$ & $1.697(0.908-3.175)$ & 0.096 \\
\hline Yes & $1(0.7)$ & $0(0.0)$ & & & 27 (20.6) & $26(30.6)$ & & \\
\hline \multicolumn{9}{|l|}{ MLH1 Methylation } \\
\hline Unmethylated & $131(96.3)$ & $64(98.5)$ & $0.409(0.047-3.577)$ & 0.405 & $62(47.3)$ & $48(56.5)$ & $0.693(0.400-1.199)$ & 0.189 \\
\hline Methylated & $5(3.7)$ & $1(1.5)$ & & & $69(52.7)$ & $37(43.5)$ & & \\
\hline \multicolumn{9}{|c|}{ BRAF V600E Mutation } \\
\hline No & $126(90.6)$ & $66(94.3)$ & $0.587(0.184-1.873)$ & 0.364 & $67(53.2)$ & $53(66.3)$ & $0.579(0.324-1.034)$ & 0.064 \\
\hline Yes & $13(9.4)$ & $4(5.7)$ & & & $59(46.8)$ & $27(33.8)$ & & \\
\hline \multicolumn{9}{|l|}{ CIMP Status } \\
\hline Negative & $91(92.9)$ & $42(93.3)$ & $0.929(0.229-3.770)$ & 0.917 & $45(52.3)$ & $34(60.7)$ & $0.710(0.359-1.406)$ & 0.325 \\
\hline Positive & $7(7.1)$ & $3(6.7)$ & & & $41(47.7)$ & $22(39.3)$ & & \\
\hline \multicolumn{9}{|l|}{ Survival Status } \\
\hline Alive & $95(65.5)$ & $50(70.4)$ & $0.782(0.424-1.444)$ & 0.432 & $91(69.5)$ & $57(67.1)$ & $1.118(0.622-2.007)$ & 0.710 \\
\hline Deceased & $50(34.5)$ & $21(29.6)$ & & & $40(30.5)$ & $28(32.9)$ & & \\
\hline
\end{tabular}

adds ratio and $95 \%$ confidence interval for methylated versus unmethylated

${ }^{\mathrm{b}} \mathrm{OR}$ and $95 \% \mathrm{Cl}$ given for lowest stage/grade versus highest stage/grade

cProximal tumor location includes lesions up to and including the splenic flexure

${ }^{\mathrm{d}}$ Mucinous histology includes the presence of any mucin within the tumor stroma 
marked by both genetic mutations and hypermethylation in CRC, acquires DNA methylation equally across subtypes.

This is the first study to investigate DNA methylation of ITF2 in CRC cases. Here, we have established that ITF2 methylation is a tumor-associated event, being a rare occurrence in normal tissue DNA. One sample out of 47 normal colonic tissue samples was methylated, but this rare occurrence may possibly be due to the field effect, or field cancerization, in which apparently normal cells acquire genetic and/or epigenetic alterations and may eventually progress to cancer. With regards to tumor methylation of ITF2, we showed that it is associated with the MSI-H phenotype. ITF2 has been reported to be a tumor suppressor that can induce cell cycle arrest and is sometimes lost due to loss of heterozygosity at 18q21 [31]. However, ITF2 expression has been found to be upregulated in some cancers with aberrantly activated Wnt signaling but decreased in others [30, 31]. Further research is required to elucidate the role of ITF2 in tumorigenesis. Treatment of gastric cancer cell lines with the DNA methyltransferase inhibitor 5-aza-2'-deoxycytidine (5-aza) restored mRNA expression in cell lines that had hypermethylation demonstrating methylationdependent regulation of this gene [33]. Thus, transcriptional silencing in CRC through methylation would likely lead to a decrease in its cellular expression levels potentially contributing to tumorigenesis.

$A P C$ promoter methylation is rarely observed in normal colonic tissue compared with CRC tumor tissue in our study population, which replicates the findings of a recent meta-analysis [44]. However, contrary to ITF2 methylation, we did not see an association with MSI-H $\mathrm{CRC}$ or any other clinical features. $A P C$ expression is at least partially regulated by DNA methylation, as its expression increases in CRC cell lines after treatment with 5-aza [45]. Several other studies have investigated the correlation between $A P C$ methylation and MSI with varying results. Studies have shown wide variation in overall APC methylation, regardless of subtype, from as low as $18 \%$ to as high as $63.4 \%[19,20]$. Our results show a more moderate level of 34-40 \% of cases methylated. Findings in the literature for the correlation between $A P C$ and MSI are even less clear, with methylation in MSI-H tumors ranging from 14.3-72.7 \% [21, 26]. However, these studies analyzed small numbers of patient samples, with a maximum of 29 MSI tumors used [24]. Our study, on the other hand, employed a total of 432 samples, 216 of which were MSI-H. This sample size is many times larger than any other of its kind, giving more statistical power and certainty to our results.

There are no differences between level of methylation at different stages of CRC diagnosis for either $A P C$ or $I T F 2$, indicating these may be early epigenetic events in tumorigenesis. Additionally, $A P C$ methylation has been detected in colon adenoma, further evidence that it is an early event [46]. Detection of $A P C$ may be further exploited as a potential biomarker by detection in other biospecimens, as its methylation has been detected in both stool and plasma [47, 48]. Further investigation of the presence of ITF2 methylation in adenomas should be undertaken, as well as whether its methylation can be detected in stool or plasma. This research will indicate the potential of utilizing ITF2 and $A P C$, perhaps in combination with other methylation markers, as noninvasive stool- or plasma-based methylation markers for CRC detection and/or subtype discrimination.

Data from colon and rectal tumors from The Cancer Genome Atlas (TCGA) shows that APC mutation rates differ among the 224 tumors sequenced by exome sequencing. TCGA data described hypermutated tumors, which have a mutation rate of $12 / 10^{6}$ and consist mostly of MSI-H tumors. The prevalence of $A P C$ mutation in these hypermutated tumors is $51 \%$ [15]. Alternatively, non-hypermutated tumors, defined by a mutation rate $<8.24 / 10^{6}$ and consisting mostly of MSS tumors, incurred $A P C$ mutations in $81 \%$ of cases [15]. This disparity in $A P C$ mutation rates may be explained by DNA methylation to inactivate $A P C$ leading to constitutive ligand-independent Wnt signaling. In this same data set ITF2 is genetically altered in only $3 \%$ of tumors, thus, methylation is likely to play a larger role in ITF2 dysregulation in cancer $[49,50]$.

While MSI-H tumors are a largely well-defined subtype of CRC, MSS tumors comprise the majority of cases and exhibit a wide variety of molecular characteristics. Thus, there is an emerging research focus to further classify molecular subtypes of CRC. Recently, four consensus molecular subtypes were defined. The first subtype consists mostly of MSI cases [14]. The remaining three subtypes are defined by 'canonical' Wnt and MYC activation, metabolic dysregulation, or mesenchymal activation. Our results indicated that some MSS cases incur methylation of the Wnt genes studied, so perhaps these cases belong to the subtype characterized by Wnt activation. It would be interesting to see which subclassification the MSS cases used in this study belong to, and how ITF2 or APC methylation profiles differ among the four subtypes.

MSI-H tumors often overlap with CIMP-positive status. Thus, the association we see between MSI-H and ITF2 methylation may in fact be part of the widespread hypermethylation of $\mathrm{CpG}$ islands that characterizes CIMP tumors. CIMP status information is unavailable for some Ontario cases and all Newfoundland cases utilized in this study, thus we do not have a complete picture of CIMP for our cohort. From our available data we did see a trend between CIMP-positive status and ITF2 methylation among MSS cases. However, there 
were only ten cases in this group. From our current findings as well as previous investigation into epigenetic regulation of Wnt signalling genes we have found that dysregulation through aberrant methylation is implicated in all subtypes of CRC, not solely in CIMP-positive cases. APC, which abrogates Wnt signaling intracellularly, is methylated in a proportion of CRCs, regardless of subtype while ITF2, a downstream target of Wnt signaling, is methylated more often in MSI-H tumors. Our lab has previously found that DKK1 and SFRP1 promoter methylation, coding for two extracellular Wnt antagonists, segregate strongly with different CRC subtypes. DKK1 methylation is associated with the MSI-H phenotype and other MSI-associated features, while SFRP1 methylation is associated with MSS tumors [34]. We also found that Wnt5a methylation, which codes for an extracellular ligand of the non-canonical Wnt pathway, is associated with MSI-H [35]. These results were found in the same cohort of Ontario and Newfoundland patients used in this study. These observations underscore the importance of both Wnt signaling and the role of DNA methylation in CRC.

One limitation of this study to bear in mind is that only a subset of available MSS cases was chosen for analysis by matching to MSI-H cases by age quartile, stage and sex. Individuals with MSI-H CRC are generally a younger age, more frequently female, have a lower tumor stage and are more frequently CIMP-positive than those individuals with MSS tumors. Thus, the MSS cases analyzed in this study do not wholly represent all MSS cases from our Ontario and Newfoundland populations. Additionally, we did not select MSS cases from the entire Ontario cohort, but only a subset available at the time this study was undertaken. The subset that we selected from did not differ in age, sex, stage or CIMP rates from the entire cohort.

The strengths of our study include large sample size, the inclusion of two independent well-characterized population-based cohorts and the choice of technology. The use of MethyLight technology is superior to methylation-specific PCR (MSP) and offers several advantages including a quantitative, high-throughput methylation-specific real-time PCR-based technique, which is amenable to using small quantities of DNA extracted from archival tissue specimens. MSP is a more qualitative and subjective method that has been used in many prior studies of $A P C$ methylation.

\section{Conclusions}

Our findings demonstrate the importance of DNA methylation in the regulation of genes selected for analysis and its differing effects based on tumor subtype. ITF2 is not yet well studied in CRC and we have now shown that this gene incurs MSI-associated hypermethylation. For $A P C$, both mutation and methylation play a role in its dysregulation. It is likely that methylation of $A P C$ plays a secondary role in
CRC to more commonly occurring mutations and may act to fine-tune Wnt signaling. With both mutation and methylation contributing to regulation of this gene, it is possible the sequence of events may dictate the way CRC evolves. Based on its high specificity for CRC, APC methylation may offer usefulness as a marker within a panel of other genes for CRC detection and ITF2 may be useful for detection of MSI-H tumors. Future studies to independently validate these findings are warranted. Overall, this study has investigated methylation of the Wnt genes $A P C$ and ITF2 in a large cohort of MSI-H and matched MSS CRC tumors to find that ITF2 methylation is significantly associated with MSI-H tumors while $A P C$ methylation is a tumor-specific event in CRC, which does not differ significantly between MSI-H and MSS subtypes or other clinicopathological variables.

\section{Abbreviations \\ 5-aza: 5-aza-2'-deoxycytidine; APC: adenomatous polyposis coli; Cl: confidence interval; CIMP: CpG island methylator phenotype; CRC: colorectal cancer; DKK1: dickkopf 1 homolog [Xenopus laevis]; ITF2: immunoglobulin transcription factor 2/transcription factor 4; MLH1: MutL homolog 1; MSI: microsatellite instability; MSI-H: microsatellite instability high; MSI-L: microsatellite instability low; MSP: methylation-specific polymerase chain reaction; MSS: microsatellite stable; NFCCR: Newfoundland Familial Colorectal Cancer Registry; OFCCR: Ontario Familial Colorectal Cancer Registry; OR: odds ratio; PMR: percent methylated reference; SD: standard deviation; SFRP1: secreted frizzled-related protein 1; TCGA: The Cancer Genome Atlas; Wnt5a: wingless-type MMTV integration site family, member $5 \mathrm{~A}$.}

\section{Competing interests}

The authors declare that they have no competing interests.

\section{Authors' contributions}

AJS carried out the methylation analysis, performed the statistical analysis, and drafted the manuscript. DD and ED participated in patient recruitment and coordination. DDB and JPY performed mutation analysis. DW performed CIMP analysis. PSP, RCG, SG, and JRM participated in study design and patient recruitment. JAK participated in the design of the study and statistical analysis and interpretation of data. BB conceived the study, contributed in analysis and interpretation of results, and drafted the manuscript. All authors read and approved the final manuscript.

\section{Acknowledgements}

This work was undertaken at the Department of Laboratory Medicine and Pathobiology at the University of Toronto, Toronto, Ontario, Canada and was conducted at the Lunenfeld-Tanenbaum Research Institute, Mount Sinai Hospital, Toronto, Ontario, Canada. We sincerely thank the investigators, staff, and participants of the Colon Cancer Family Registry for their dedicated contributions leading to this work. We gratefully acknowledge The Jeremy Jass Memorial Pathology Bank for the tissue samples and pathology data used in this study. This work was supported by grant UM1 CA167551 from the National Cancer Institute and through cooperative agreements with the following CCFR centers: Ontario Registry for Studies of Familial Colorectal Cancer (U01/U24 CA074783) and Australasian Colorectal Cancer Family Registry (U01/U24 CA097735). This work was also supported by a Team Grant from the Canadian Institutes of Health Research (CTP-79845) awarded to BB, JAK, SG, RCG, and PSP by the NCI under Request For Applications (CA-95-011). The content of this manuscript does not necessarily reflect the views or policies of the National Cancer Institute or any of the collaborating centers in the Colon Cancer Family Registry (CCFR), nor does mention of trade names, commercial products, or organizations imply endorsement by the U.S. Government or CFR. AJS was supported by the Interdisciplinary Health Research Team Program studentship funded by the Canadian Institutes of Health Research, the Lunenfeld-Tanenbaum Research Institute Studentship at Mount Sinai Hospital, and the University of Toronto Fellowship award. 
The funders had no role in study design, data collection and analysis, decision to publish, or preparation of the manuscript.

\begin{abstract}
Author details
'Department of Laboratory Medicine and Pathobiology, University of Toronto, Toronto, ON, Canada. ${ }^{2}$ Lunenfeld-Tanenbaum Research Institute of Mount Sinai Hospital, Toronto, ON, Canada. ${ }^{3}$ Ontario Familial Colorectal Cancer Registry, Toronto, ON, Canada. ${ }^{4}$ Faculty of Medicine, Memorial University of Newfoundland, St John's, Newfoundland, Canada. ${ }^{5}$ Oncogenomics Group, Genetic Epidemiology Laboratory, Department of Pathology, The University of Melbourne, Parkville, VIC, Australia. ${ }^{6}$ Centre for Epidemiology and Biostatistics, Melbourne School of Population and Global Health, The University of Melbourne, Parkville, VIC, Australia. 'Department of Haematology and Oncology, The Queen Elizabeth Hospital, Woodville, South Australia, Australia. ${ }^{8}$ USC Epigenome Center, University of Southern California, Los Angeles, CA, USA. 'Department of Surgery, University of Toronto, Toronto, ON, Canada. ${ }^{10}$ Dalla Lana School of Public Health, University of Toronto, Toronto, ON, Canada. ${ }^{11}$ Department of Pathology, University Health
\end{abstract} Network, Toronto, ON, Canada.

\section{Received: 10 August 2015 Accepted: 8 February 2016}

\section{Published online: 17 February 2016}

\section{References}

1. Siegel R, Naishadham D, Jemal A. Cancer Statistics, 2013. CA Cancer J Clin. 2011;60:69-90

2. Moiel D, Thompson J. Early detection of colon cancer - the Kaiser Permanente Northwest 30-year history: how do we measure success? Is it the test, the number of tests, the stage, or the percentage of screen-detected patients? Perm J. 2011:15:30-8.

3. Shima K, Morikawa T, Yamauchi M, Kuchiba A, Imamura Y, Liao X, et al. TGFBR2 and BAX mononucleotide tract mutations, microsatellite instability, and prognosis in 1072 colorectal cancers. PLoS One. 2011;6:e25062.

4. Boland CR, Thibodeau SN, Hamilton SR, Sidransky D, Eshleman JR, Burt RW et al. A National Cancer Institute Workshop on Microsatellite Instability for cancer detection and familial predisposition: development of international criteria for the determination of microsatellite instability in colorectal cancer. Cancer Res. 1998;58:5248-57.

5. Moslein G, Tester DJ, Lindor NM, Honchel R, Cunningham JM, French AJ, et al. Microsatellite instability and mutation analysis of hMSH2 and hMLH1 in patients with sporadic, familial and hereditary colorectal cancer. Hum Mol Genet. 1996:5:1245-52.

6. Grady WM. Genomic instability and colon cancer. Cancer Metastasis Rev. 2004;23:11-27.

7. Hong SP, Min BS, Kim TI, Cheon JH, Kim NK, Kim H, et al. The differential impact of microsatellite instability as a marker of prognosis and tumor response between colon cancer and rectal cancer. Eur J Cancer. 2012;48:1235-43.

8. Michel S, Benner A, Tariverdian M, Wentzensen N, Hoefler P, Pommerencke $\mathrm{T}$, et al. High density of FOXP3-positive T cells infiltrating colorectal cancers with microsatellite instability. Br J Cancer. 2008;99:1867-73.

9. Goldstein J, Tran B, Ensor J, Gibbs P, Wong HL, Wong SF, et al. Multicenter retrospective analysis of metastatic colorectal cancer with high-level microsatellite instability (MSI-H). Ann Oncol. 2014:25:1032-8.

10. Toyota M, Ahuja N, Ohe-Toyota M, Herman JG, Baylin SB, Issa JP. CpG island methylator phenotype in colorectal cancer. Proc Natl Acad Sci U S A. 1999; 96:8681-6.

11. Phipps Al, Limburg PJ, Baron JA, Burnett-Hartman AN, Weisenberger DJ, Laird PW, et al. Association between molecular subtypes of colorectal cancer and patient survival. Gastroenterology. 2015;148:77-87.

12. Shen $L$, Catalano PJ, Benson AB, O'Dwyer P, Hamilton SR, Issa JP. Association between DNA methylation and shortened survival in patients with advanced colorectal cancer treated with 5-fluorouracil based chemotherapy. Clin Cancer Res. 2007:13:6093-8.

13. Ogino S, Nosho K, Kirkner GJ, Kawasaki T, Meverhardt JA, Loda M, et al. CpG island methylator phenotype, microsatellite instability, BRAF mutation and clinical outcome in colon cancer. Gut. 2009:58:90-6.

14. Guinney J, Dienstmann R, Wang X, de Reyniès A, Schlicker A, Soneson C, et al. The consensus molecular subtypes of colorectal cancer. Nat Med. 2015;21:1350-6.

15. Muzny DM, Bainbridge MN, Chang K, Dinh HH, Drummond JA, Fowler G, et al. Comprehensive molecular characterization of human colon and rectal cancer. Nature. 2012;487:330-7.
16. Gregorieff A, Clevers $H$. Wht signaling in the intestinal epithelium: from endoderm to cancer. Genes Dev. 2005;19:877-90.

17. Huelsken J, Birchmeier W. New aspects of Wnt signaling pathways in higher vertebrates. Curr Opin Genet Dev. 2001:11:547-53.

18. Willert K, Jones KA. Wnt signaling: is the party in the nucleus? Genes Dev. 2006; 20:1394-404.

19. Chen J, Rocken C, Lofton-Day C, Schulz HU, Muller O, Kutzner N, et al. Molecular analysis of APC promoter methylation and protein expression in colorectal cancer metastasis. Carcinogenesis. 2005;26:37-43.

20. Chen SP, Chiu SC, Wu CC, Lin SZ, Kang JC, Chen YL, et al. The association of methylation in the promoter of APC and MGMT and the prognosis of Taiwanese CRC patients. Genet Test Mol Biomarkers. 2009;3:67-71.

21. Derks S, Postma C, Caralho B, van den Bosch SM, Moerkerk PT, Herman $J G$, et al. Integrated analysis of chromosomal, microsatellite and epigenetic instability in colorectal cancer identifies specific associations between promoter methylation of pivotal tumor suppressor and DNA repair genes and specific chromosomal alterations. Carcinogenesis. 2008;29:434-9.

22. Kumar K, Brim H, Giardiello F, Smoot DT, Nouraie M, Lee EL, et al. Distinct BRAF (V600E) and KRAS mutations in high microsatellite instability sporadic colorectal cancer in African Americans. Clin Cancer Res. 2009;15:1155-61.

23. Naghibalhossaini F, Zamani M, Mokarram P, Khalili I, Rasti M, Mostafavi-Pour Z. Epigenetic and genetic analysis of Wnt signaling pathway in sporadic colorectal cancer patients from Iran. Mol Biol Rep. 2012;39:6171-8.

24. Gay LJ, Mitrou PN, Keen J, Bowman R, Naguib A, Cooke J, et al. Dietary, lifestyle and clinicopathological factors associated with APC mutations and promoter methylation in colorectal cancers from the EPIC-Norfolk study. J Pathol. 2012; 228:405-15.

25. Goel A, Nagasaka T, Arnold CN, Inoue T, Hamilton C, Niedzwiecki D, et al. The CpG island methylator phenotype and chromosomal instability are inversely correlated in sporadic colorectal cancer. Gastroenterology. 2007;132:127-38.

26. Kim JC, Choi JS, Roh SA, Cho DH, Kim TW, Kim YS. Promoter methylation of specific genes is associated with the phenotype and progression of colorectal adenocarcinomas. Ann Surg Oncol. 2010;17:1767-76.

27. Thorstensen L, Lind GE, Lovig T, Diep CB, Meling Gl, Rognum TO, et al. Genetic and epigenetic changes of components affecting the WNT pathway in colorectal carcinomas stratified by microsatellite instability. Neoplasia. 2005;7:99-108.

28. lacopetta B, Grieu F, Li W, Ruszkiewicz A, Caruso M, Moore J, et al. APC gene methylation is inversely correlated with features of the $\mathrm{CpG}$ island methylator phenotype in colorectal cancer. Int J Cancer. 2006;119:2272-8.

29. Zhai Y, Wu R, Schwartz DR, Darrah D, Reed H, Kolligs FT, et al. Role of beta-catenin/T-cell factor-regulated genes in ovarian endometrioid adenocarcinomas. Am J Pathol. 2002;160:1229-38.

30. Kolligs FT, Nieman MT, Winer I, Hu G, Van Mater D, Feng Y, et al. ITF-2, a downstream target of the Wnt/TCF pathway, is activated in human cancers with $\beta$-catenin defects and promotes neoplastic transformation. Cancer Cell. 2002;1:145-55.

31. Herbst A, Helferich S, Behrens A, Goke B, Kolligs FT. The transcription factor ITF-2A induces cell cycle arrest via p21(Cip1). Biochem Biophys Res Commun. 2009;387: 736-40.

32. Joo JK, Kim SH, Kim HG, Kim DY, Ryu SY, Lee KH, et al. Methylation of transcription factor 4 in gastric carcinoma. Ann Surg Oncol. 2010;17: 3344-53.

33. Kim JH, Kim M, Noh SM, Song KS, Kang GH, Kim HJ, et al. CpG methylation in exon 1 of transcription factor 4 increases with age in normal gastric mucosa and is associated with gene silencing in intestinal-type gastric cancers. Carcinogenesis. 2008:29:1623-31.

34. Rawson JB, Manno M, Mrkonjic M, Daftary D, Dicks E, Buchanan DD, et al. Promoter methylation of Wnt antagonists DKK1 and SFRP1 is associated with opposing tumor subtypes in two large populations of colorectal cancer patients. Carcinogenesis. 2011;32:741-7.

35. Rawson JB, Mrkonjic M, Daftary D, Dicks E, Buchanan DD, Younghusband $H B$, et al. Promoter methylation of Wnt5a is associated with microsatellite instability and BRAF V600E mutation in two large populations of colorectal cancer patients. Br J Cancer. 2011;104:1906-12.

36. Cotterchio M, McKeown-Eyssen G, Sutherland H, Buchan G, Aronson M, Easson AM, et al. Ontario familial colon cancer registry: methods and first-year response rates. Chron Dis Can. 2000;21:81-6.

37. Green RC, Green JS, Buehler SK, Robb JD, Daftary D, Gallinger S, et al. Very high incidence of familial colorectal cancer in Newfoundland: a comparison with Ontario and 13 other population-based studies. Fam Cancer. 2007;6:53-62. 
38. Woods MO, Hyde AJ, Curtis FK, Stuckless S, Green JS, Pollett AF, et al. High frequency of hereditary colorectal cancer in Newfoundland likely involves novel susceptibility genes. Clin Cancer Res. 2005;11:6853-61.

39. Lindor NM, Burgart LJ, Leontovich O, Goldberg RM, Cunningham JM, Sargent DJ, et al. Immunohistochemistry versus microsatellite instability testing in phenotyping colorectal tumors. J Clin Oncol. 2002;20:1043-8.

40. Weisenberger DJ, Campan M, Long TI, Kim M, Woods C, Fiala E, et al. Analysis of repetitive element DNA methylation by MethyLight. Nucleic Acids Res. 2005; 33:6823-36.

41. Campan M, Weisenberger DJ, Trinh B, Laird PW. MethyLight. Methods Mol Biol. 2009:507:325-37.

42. Mrkonjic M, Roslin NM, Greenwood CM, Raptis S, Pollett A, Laird PW, et al. Specific variants in the MLH1 gene region may drive DNA methylation, loss of protein expression, and MSI-H colorectal cancer. PLoS One. 2010;5:e13314.

43. Weisenberger DJ, Levine AJ, Long TI, Buchanan DD, Walters R, Clendenning $M$, et al. Association of the colorectal CpG island methylator phenotype with molecular features, risk factors, and family history. Cancer Epidemiol Biomarkers Prev. 2015;24:512-9.

44. Ding Z, Jiang T, Piao Y, Han T, Han Y, Xie X. Meta-analysis of the association between APC promoter methylation and colorectal cancer. Onco Targets Ther. 2015;8:211-22.

45. Deng G, Song GA, Pong E, Sleisenger M, Kim YS. Promoter methylation inhibits APC gene expression by causing changes in chromatin conformation and interfering with the binding of transcription factor CCAAT-binding factor. Cancer Res. 2004;64:2692-8.

46. Yang Q, Dong Y, Wu W, Zhu C, Chong H, Lu J, et al. Detection and differential diagnosis of colon cancer by a cumulative analysis of promoter methylation. Nat Commun. 2012;3:1206.

47. Azuara D, Rodriguez-Moranta F, de Oca J, Soriano-Izguierdo A, Mora J, Guardiola $J$, et al. Novel methylation panel for the early detection of colorectal tumors in stool DNA. Clin Colorectal Cancer. 2010;9:168-76.

48. Lee BB, Lee El, Jung EH, Chun HK, Chang DK, Song SY, et al. Aberrant methylation of APC, MGMT, RASSF2A, and WIF-1 genes in plasma as a biomarker for early detection of colorectal cancer. Clin Cancer Res. 2009;15:6185-91.

49. Gao J, Aksoy BA, Dogrusoz U, Dresdner G, Gross B, Sumer SO, et al. Integrative analysis of complex cancer genomics and clinical profiles using cBioPortal. Sci Signal. 2013;6:pl1.

50. Cerami E, Gao J, Dogrusoz U, Gross BE, Sumer SO, Aksoy BA, et al. The cBio cancer genomics portal: an open platform for exploring multidimensional cancer genomics data. Cancer Discov. 2012;2:401-4.

\section{Submit your next manuscript to BioMed Central and we will help you at every step:}

- We accept pre-submission inquiries

- Our selector tool helps you to find the most relevant journal

- We provide round the clock customer support

- Convenient online submission

- Thorough peer review

- Inclusion in PubMed and all major indexing services

- Maximum visibility for your research

Submit your manuscript at www.biomedcentral.com/submit
Biomed Central 